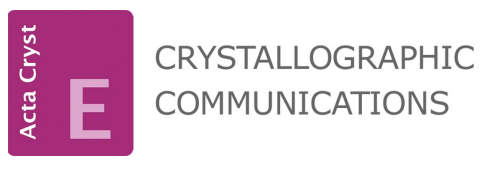

ISSN 2056-9890

Received 7 February 2018

Accepted 10 February 2018

Edited by M. Zeller, Purdue University, USA

Keywords: crystal structure; Schiff bases; molecular conformation; hydrogen bonding; supramolecular assembly.

CCDC reference: 1823227

Supporting information: this article has supporting information at journals.iucr.org/e

\section{Reinvestigation of the crystal structure of $\mathrm{N}$-(4- chlorobenzylidene)-2-hydroxyaniline: a three- dimensional structure containing $\mathrm{O}-\mathrm{H} \cdots \mathrm{N}, \mathrm{O}-$ $\mathrm{H} \cdots \mathrm{O}$ and $\mathrm{C}-\mathrm{H} \cdots \pi($ arene) hydrogen bonds}

\author{
Marisiddaiah Girisha, ${ }^{a}$ Hemmige S. Yathirajan, ${ }^{a}$ Ravindranath S. Rathore ${ }^{b}$ and \\ Christopher Glidewell
}

${ }^{\mathbf{a}}$ Department of Studies in Chemistry, University of Mysore, Manasagangotri, Mysuru 570 006, India, ${ }^{\mathbf{b}}$ Centre for Biological Sciences (Bioinformatics), School of Earth, Biological and Environmental Sciences, Central University of South Bihar, Patna 800 014, India, and ${ }^{\mathbf{C}}$ School of Chemistry, University of St Andrews, St Andrews, Fife KY16 9ST, Scotland. *Correspondence e-mail: giri.viji.shiva@gmail.com

The molecule of the title compound, $\mathrm{C}_{13} \mathrm{H}_{10} \mathrm{ClNO}$, (I), which contains an intramolecular $\mathrm{O}-\mathrm{H} \cdots \mathrm{N}$ hydrogen bond, is almost planar: the dihedral angle between the two aryl rings is only $3.31(9)^{\circ}$. The molecules of (I) are linked into sheets by two $\mathrm{C}-\mathrm{H} \cdots \pi$ (arene) hydrogen bonds and the sheets are linked into a three-dimensional structure by $\mathrm{O}-\mathrm{H} \cdots \mathrm{O}$ hydrogen bonds. Comparisons are made with the structures of a number of related compounds.

\section{Chemical context}

Schiff bases exhibit a very wide range of biological activities (da Silva et al., 2011) and are also of interest because of the photochromic and thermochromic properties (Hadjoudis \& Mavridis, 2004; Minkin et al., 2011). The molecular structure of $\mathrm{N}$-(4-chlorobenzylidene)-2-hydroxyaniline (I) was reported in the space group $P 2_{1} / n$ a number of years ago [CSD (Groom $e t$ al., 2016) refcode FAKDIE; Kamwaya \& Khoo, 1985]. However, scrutiny of the reported structure reveals a number of unexpected features: the refinement was conducted in a non-standard monoclinic cell having $\beta<90^{\circ}$; the $\mathrm{C}-\mathrm{C}$ distances in the aryl rings range between 1.336 and $1.427 \AA$; no $\mathrm{H}$ atoms bonded to $\mathrm{C}$ atoms were included; and the $\mathrm{C}-\mathrm{O}-\mathrm{H}$ angle was reported as $88^{\circ}$, which seems very small, while the associated intramolecular $\mathrm{H} \cdots \mathrm{N}$ distance was only $1.66 \AA$, which is very short, even for a strong $\mathrm{O}-\mathrm{H} \cdots \mathrm{N}$ hydrogen bond. Hence any conclusions drawn from the deposited atomic coordinates may be untrustworthy. The structures of several positional isomers of (I) have been reported recently (Kazak et al., 2004; Sundararaman et al., 2007; Saranya et al., 2015) and in view of these reports and of the widespread applications of Schiff bases, we have accordingly now collected a new data set for compound (I), whose structure we report here (Fig. 1).

\section{Structural commentary}

The molecular skeleton of compound (I) (Fig. 1) is very nearly planar: the r.m.s. deviation from the mean plane through all of the non-H atoms is only $0.043 \AA$, with a maximum displacement from this plane of 0.0900 (10) $\AA$ for atom Cl14. The 
dihedral angle between the two aryl rings in the molecule of (I) is only $3.31(9)^{\circ}$. A fairly short intramolecular $\mathrm{O}-\mathrm{H} \cdots \mathrm{N}$ contact (Table 1) may be an influence on the molecular conformation. The $\mathrm{C}-\mathrm{C}$ distances within the rings lie in the range 1.377 (3)-1.393 (3) $\AA$ for the hydroxylated ring, and 1.366 (3)-1.387 (3) $\AA$ for the chlorinated ring, much smaller than the range previously reported (Kamwaya \& Khoo, 1985), while the $\mathrm{C}-\mathrm{O}-\mathrm{H}$ angle is $103.0(18)^{\circ}$. The inter-axial angle $\beta$, as found here and as previously reported, $\beta^{\prime}$, are related by $\beta=\left(180-\beta^{\prime}\right)$ and the atomic coordinates found here can be related to those reported previously, after inversion and a straightforward origin shift, by the transformation $(x, y,-z)$, suggesting that the previous determination may have in advertently used a left-handed axis set.

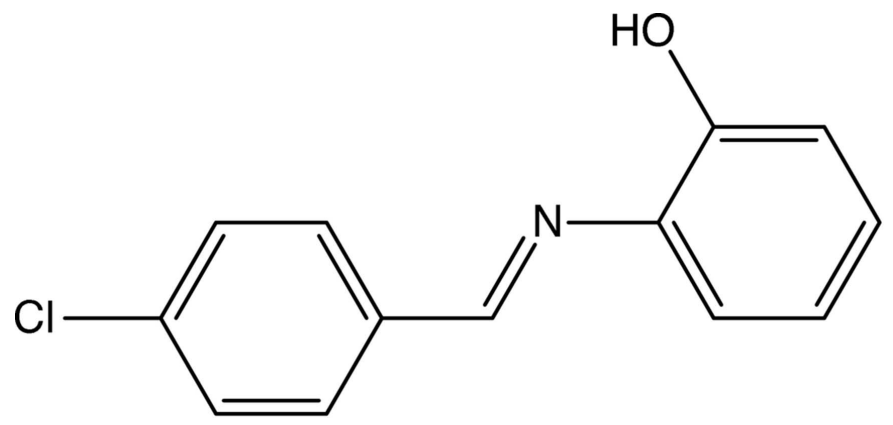

\section{Supramolecular features}

The supramolecular assembly is dominated by two $\mathrm{C}-$ $\mathrm{H} \cdots \pi$ (arene) hydrogen bonds (Table 1 ): that having atom $\mathrm{C} 6$ as the donor links molecules related by the $2_{1}$ screw axis along $(0.75, y, 0.75)$, and that having atom $\mathrm{C} 15$ as the donor links molecules related by the $2_{1}$ screw axis along $(0.25, y, 0.75)$, so forming two distinct types of chain parallel to [010]. In the first of these, the chlorinated ring provides both the donor and the acceptor, while in the second the hydroxylated ring provides both the donor and the acceptor (Fig. 2). The combination of these two chains links the molecules of (I) into sheets lying parallel to (001) (Fig. 2). Two sheets of this type, related to one another by inversion, pass through each unit cell, in the domains $0<z<0.5$ and $0.5<z<1.0$. Adjacent sheets are

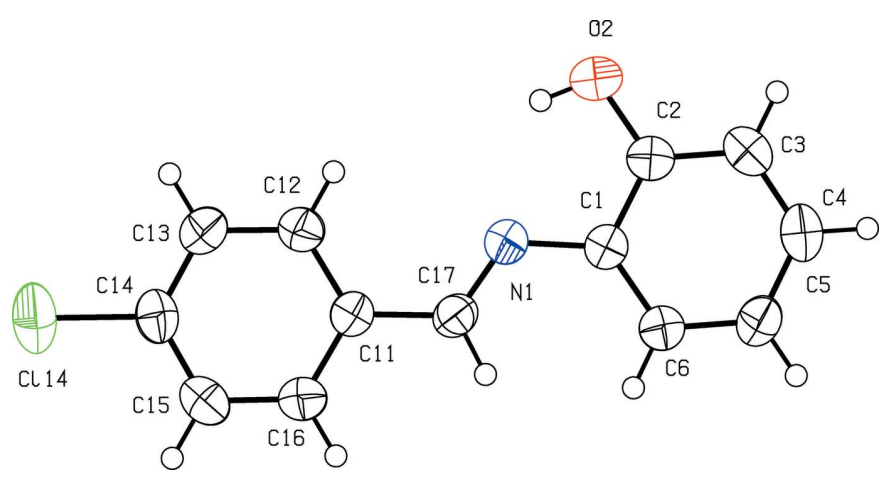

Figure 1

The molecular structure of compound (I), showing the atom-labelling scheme. Displacement ellipsoids are drawn at the $50 \%$ probability level.
Table 1

Hydrogen-bond geometry $\left(\AA,^{\circ}\right)$.

$C g 1$ and $C g 2$ are the centroids of the $\mathrm{C} 1-\mathrm{C} 6$ and $\mathrm{C} 11-\mathrm{C} 16$ rings, respectively.

\begin{tabular}{lllll}
\hline$D-\mathrm{H} \cdots A$ & $D-\mathrm{H}$ & $\mathrm{H} \cdots A$ & $D \cdots A$ & $D-\mathrm{H} \cdots A$ \\
\hline $\mathrm{O} 2-\mathrm{H} 2 \cdots \mathrm{N} 1$ & $0.84(3)$ & $2.05(3)$ & $2.626(2)$ & $125(2)$ \\
$\mathrm{O} 2-\mathrm{H} 2 \cdots \mathrm{O} 2^{\mathrm{i}}$ & $0.84(3)$ & $2.44(3)$ & $2.899(2)$ & $115(2)$ \\
$\mathrm{C} 6-\mathrm{H} 6 \cdots C g 1^{\mathrm{ii}}$ & 0.93 & 2.79 & $3.491(2)$ & 133 \\
$\mathrm{C} 15-\mathrm{H} 15 \cdots C g 2^{\mathrm{iii}}$ & 0.93 & 2.96 & $3.675(2)$ & 135 \\
\hline
\end{tabular}

Symmetry codes: (i) $\quad-x+1,-y+2,-z+1$; (ii) $\quad-x+\frac{3}{2}, y-\frac{1}{2},-z+\frac{3}{2}$; $\quad$ (iii) $-x+\frac{1}{2}, y-\frac{1}{2},-z+\frac{3}{2}$.

linked into a continuous three-dimensional framework by a combination of a short $\mathrm{O}-\mathrm{H} \cdots \mathrm{O}$ contact involving inversionrelated pairs of molecules (Fig. 3), and an aromatic $\pi-\pi$ stacking interaction. The aryl rings $(\mathrm{C} 1-\mathrm{C} 6)$ and $(\mathrm{C} 11-\mathrm{C} 16)$ in the molecules at $(x, y, z)$ and $(1-x, 1-y, 1-z)$, respectively, which lie in adjacent sheets, make a dihedral angle of $3.31(9)^{\circ}$ : the ring centroid separation is 3.773 (2) $\AA$ and the shortest perpendicular distance from the centroid of one ring to the plane of the other is 3.465 (2) $\AA$, giving a ring centroid offset of $c a 1.49 \AA$. In the earlier report (FAKDIE; Kamwaya \&

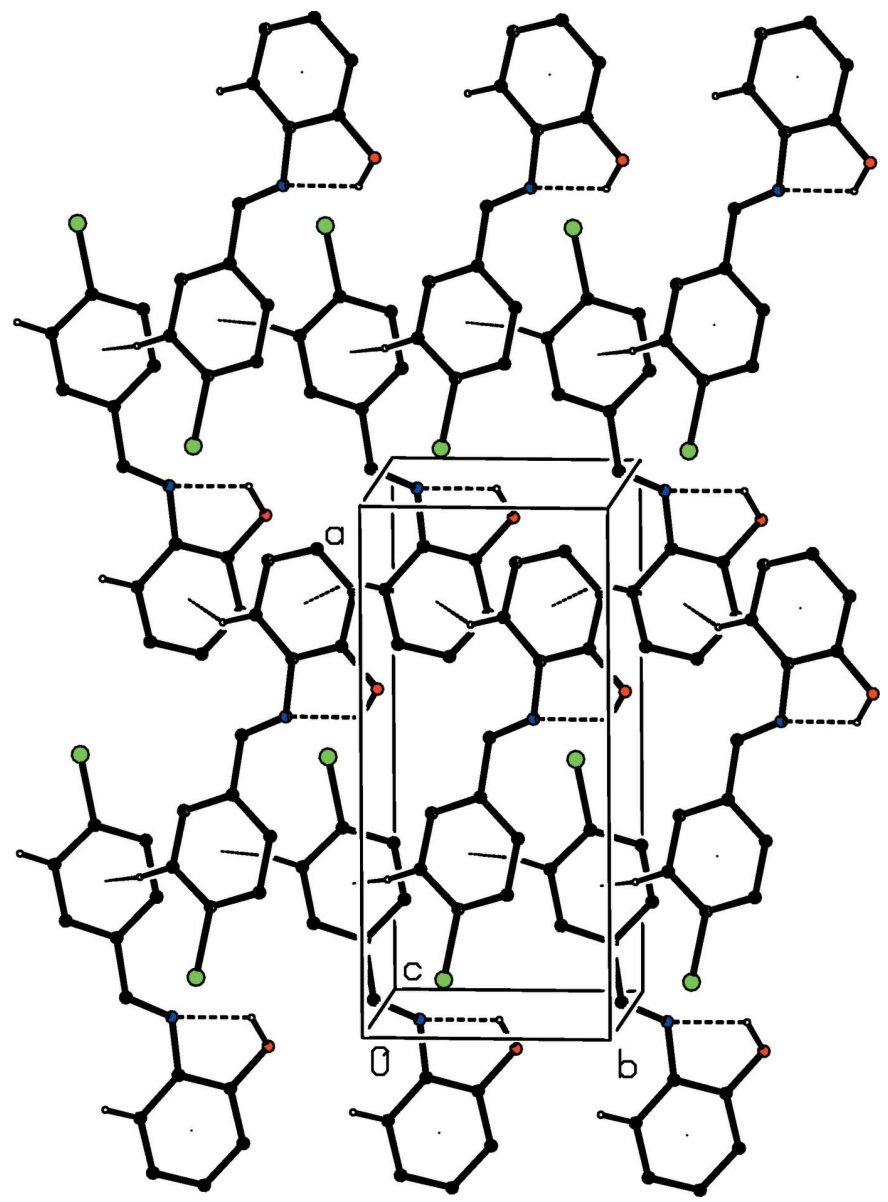

Figure 2

Part of the crystal structure of compound (I), showing the formation of a sheet lying parallel to (001) and built from $\mathrm{C}-\mathrm{H} \cdots \pi$ (arene) hydrogen bonds. For the sake of clarity, $\mathrm{H}$ atoms bonded to $\mathrm{C}$ atoms but not involved in the motifs shown have been omitted. 


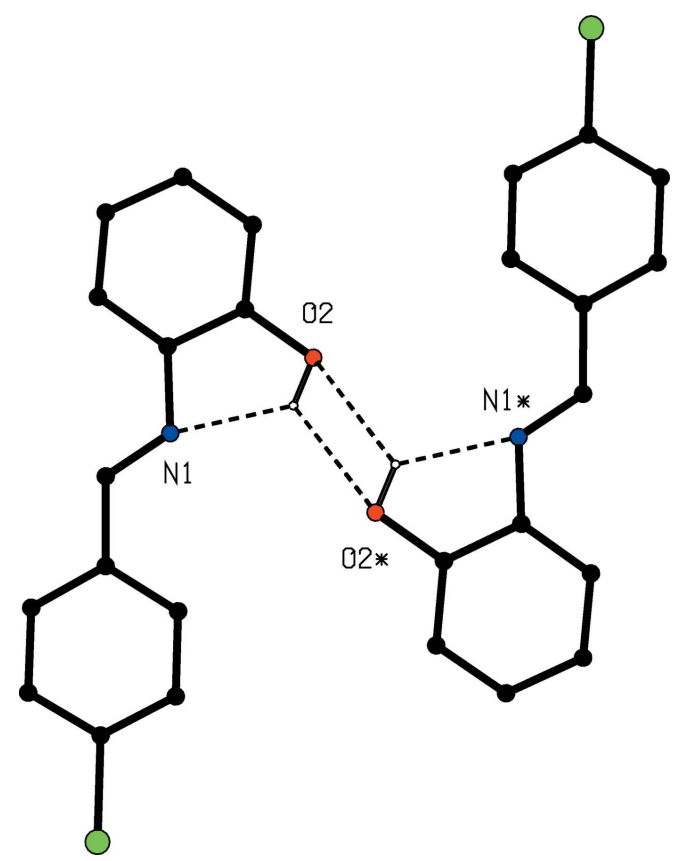

Figure 3

Part of the crystal structure of compound (I), showing the $\mathrm{O}-\mathrm{H} \cdots \mathrm{O}$ interaction between an inversion-related pair of molecules. For the sake of clarity, the unit-cell outline and $\mathrm{H}$ atoms bonded to $\mathrm{C}$ atoms have been omitted. Atoms marked with an asterisk (*) are at the symmetry position $(1-x, 2-y, 1-z)$.

Khoo, 1985), the absence of any $\mathrm{H}$ atoms bonded to $\mathrm{C}$ atoms means that the $\mathrm{C}-\mathrm{H} \cdots \pi$ (Arene) interactions were necessarily overlooked, and the apparent misplacement of the hydroxyl $\mathrm{H}$ atom noted above means that the intermolecular $\mathrm{O}-\mathrm{H} \cdots \mathrm{O}$ hydrogen bond was also overlooked.

\section{Database survey}

The structures of a number of Schiff bases which are isomeric with compound (I) have been reported in recent years (see Fig. 4). In each of compounds (II) (Kazak et al., 2004) and (III) (Sundararaman et al., 2007), the molecules are linked by O$\mathrm{H} \cdots \mathrm{N}$ hydrogen bonds to form chains of the $C(7)$ and $C(8)$ types, respectively, while in compound (IV) (Saranya et al., $2015)$ the sole $\mathrm{O}-\mathrm{H} \cdots \mathrm{N}$ interaction is intramolecular. The bromo derivative (V) (Jiao et al., 2006) is isomorphous with the chloro analogue (I), but these two compounds are not strictly isostructural in that the structure of (V) contains only one $\mathrm{C}-\mathrm{H} \cdots \pi$ (arene) hydrogen bond, as compared with two such bonds in the structure of (I). On the other hand, compounds (III) and (VI) (Jothi et al., 2012) do appear to be isostructural. Finally, we note the isomeric nitrone (VII), which crystallizes in space group $P \overline{1}$ with $Z^{\prime}=2$ : each of the two types of molecule forms a $C(4)$ chain built from $\mathrm{C}-\mathrm{H} \cdots \mathrm{O}$ hydrogen bonds (Vijayalakshmi et al., 2000).

\section{Synthesis and crystallization}

To a solution of 2-aminophenol $(0.917 \mathrm{mmol})$ in ethanol $\left(20 \mathrm{~cm}^{3}\right)$, an equimolar quantity of 4-chlorobenzaldehyde was
Table 2

Experimental details.

\begin{tabular}{|c|c|}
\hline \multicolumn{2}{|l|}{ Crystal data } \\
\hline Chemical formula & $\mathrm{C}_{13} \mathrm{H}_{10} \mathrm{ClNO}$ \\
\hline$M_{\mathrm{r}}$ & 231.67 \\
\hline Crystal system, space group & Monoclinic, $P 2_{1} / n$ \\
\hline Temperature $(\mathrm{K})$ & 296 \\
\hline$a, b, c(\AA)$ & $13.0830(17), 5.8746(6), 14.825(2)$ \\
\hline$\beta\left({ }^{\circ}\right)$ & $101.521(4)$ \\
\hline$V\left(\mathrm{~A}^{3}\right)$ & $1116.5(2)$ \\
\hline$Z$ & 4 \\
\hline Radiation type & Мо $K \alpha$ \\
\hline$\mu\left(\mathrm{mm}^{-1}\right)$ & 0.32 \\
\hline Crystal size $(\mathrm{mm})$ & $0.24 \times 0.22 \times 0.14$ \\
\hline \multicolumn{2}{|l|}{ Data collection } \\
\hline Diffractometer & Bruker APEXII \\
\hline Absorption correction & $\begin{array}{l}\text { Multi-scan ( } S A D A B S ; \text { Bruker, } \\
\text { 2012) }\end{array}$ \\
\hline$T_{\min }, T_{\max }$ & $0.897,0.957$ \\
\hline $\begin{array}{l}\text { No. of measured, independent and } \\
\text { observed }[I>2 \sigma(I)] \text { reflections }\end{array}$ & $11962,2565,1556$ \\
\hline$R_{\text {int }}$ & 0.032 \\
\hline$(\sin \theta / \lambda)_{\max }\left(\AA^{-1}\right)$ & 0.650 \\
\hline \multicolumn{2}{|l|}{ Refinement } \\
\hline$R\left[F^{2}>2 \sigma\left(F^{2}\right)\right], w R\left(F^{2}\right), S$ & $0.042,0.106,1.02$ \\
\hline No. of reflections & 2565 \\
\hline No. of parameters & 148 \\
\hline H-atom treatment & $\begin{array}{l}\mathrm{H} \text { atoms treated by a mixture of } \\
\text { independent and constrained } \\
\text { refinement }\end{array}$ \\
\hline$\Delta \rho_{\max }, \Delta \rho_{\min }\left(\mathrm{e} \AA^{-3}\right)$ & $0.16,-0.21$ \\
\hline
\end{tabular}

Computer programs: APEX2 and SAINT (Bruker, 2012), SHELXT2014 (Sheldrick, 2015a), SHELXL2014 (Sheldrick, 2015b) and PLATON (Spek, 2009).

added dropwise, with constant stirring, in the presence of a catalytic amount of glacial acetic acid. The mixture was then heated under reflux for $4 \mathrm{~h}$. When the reaction was complete, as judged using thin layer chromatography, the reaction mixture was cooled to ambient temperature and the resulting solid product was collected by filtration and recrystallized from dimethyl sulfoxide, to give crystals suitable for singlecrystal X-ray diffraction; m.p. 358 K.
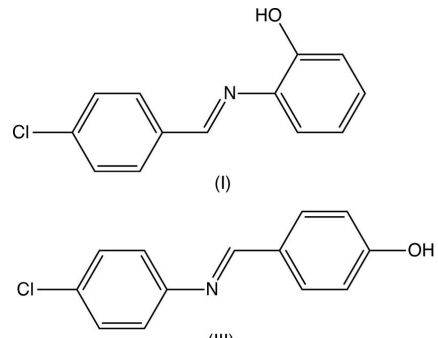

(III)

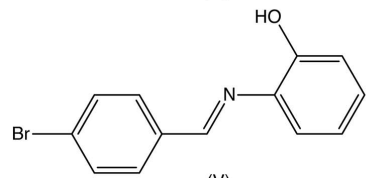

(V)
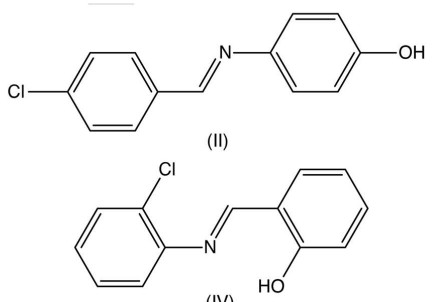

(IV)

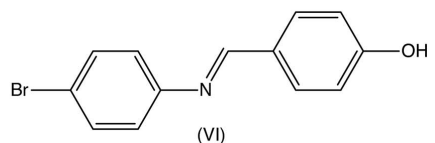

(VI)

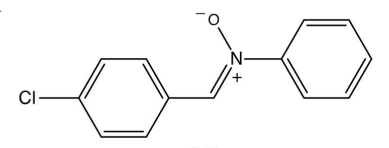

Figure 4

(VII)

Compound (I) and some closely related analogues. 


\section{Refinement}

Crystal data, data collection and structure refinement details are summarized in Table 2. All $\mathrm{H}$ atoms were located in difference maps. The $\mathrm{H}$ atoms bonded to $\mathrm{C}$ atoms were subsequently treated as riding atoms in geometrically idealized positions with $\mathrm{C}-\mathrm{H}$ distances of $0.93 \AA$ and with $U_{\text {iso }}(\mathrm{H})$ $=1.2 U_{\text {eq }}(\mathrm{C})$. For the $\mathrm{H}$ atom bonded to the $\mathrm{O}$ atom, the atomic coordinates were refined with $U_{\text {iso }}(\mathrm{H})=1.5 U_{\text {eq }}(\mathrm{O})$, giving an $\mathrm{O}-\mathrm{H}$ distance of 0.84 (3) $\AA$. In the final analysis of variance there was a negative value, -3.134 , of $K=\left[\operatorname{mean}\left(F_{o}{ }^{2}\right) /\right.$ mean $\left.\left(F_{\mathrm{c}}{ }^{2}\right)\right]$ for the group of 291 very weak reflections having $F_{\mathrm{c}} / F_{\mathrm{c}}(\max )$ in the range $0.000<F_{\mathrm{c}} / F_{\mathrm{c}}(\max )<0.003$ : this is probably a statistical artefact.

\section{Acknowledgements}

HSY thanks the University of Mysore for research facilities.

\section{Funding information}

MG thanks the UGC (India) for the award of a Rajeev Gandhi fellowship.

\section{References}

Bruker (2012). APEX2, SAINT and SADABS. Bruker AXS Inc., Madison, Wisconsin, USA.

Groom, C. R., Bruno, I. J., Lightfoot, M. P. \& Ward, S. C. (2016). Acta Cryst. B72, 171-179.

Hadjoudis, E. \& Mavridis, I. M. (2004). Chem. Soc. Rev. 33, 579-588.

Jiao, Y.-H., Zhang, Q. \& Ng, S. W. (2006). Acta Cryst. E62, o3614o3615.

Jothi, L., Vasuki, G., Babu, R. R. \& Ramamurthi, K. (2012). Acta Cryst. E68, o772.

Kamwaya, M. E. \& Khoo, L. E. (1985). J. Fiz. Malaysia, 6, 135-139.

Kazak, C., Aygün, M., Turgut, G., Odabaşoğlu, M., Büyükgüngör, O. \& Kahveci, N. (2004). Acta Cryst. E60, o252-0253.

Minkin, V. I., Tsukanov, A. V., Dubonosov, A. D. \& Bren, V. A. (2011). J. Mol. Struct. 998, 179-191.

Saranya, M., Subashini, A., Arunagiri, C. \& Muthiah, P. T. (2015). Acta Cryst. E71, o48.

Sheldrick, G. M. (2015a). Acta Cryst. A71, 3-8.

Sheldrick, G. M. (2015b). Acta Cryst. C71, 3-8.

Silva, C. M. da, da Silva, D. L., Modolo, L. V., Alves, R. B., de Resende, M. A., Martins, C. V. B., de Fátima, A. \& Ângelo, (2011). J. Adv. Res. 2, 1-8.

Spek, A. L. (2009). Acta Cryst. D65, 148-155.

Sundararaman, L., Kandasamy, R., Stoeckli-Evans, H. \& Gopalsamy, V. (2007). Acta Cryst. E63, o4805.

Vijayalakshmi, L., Parthasarathi, V. \& Manishanker, P. (2000). Acta Cryst. C56, e403-e404. 


\section{supporting information}

Acta Cryst. (2018). E74, 376-379 [https://doi.org/10.1107/S205698901800244X]

Reinvestigation of the crystal structure of $\mathrm{N}$-(4-chlorobenzylidene)-2-hydroxyaniline: a three-dimensional structure containing $\mathrm{O}-\mathrm{H} \cdots \mathrm{N}, \mathrm{O}-\mathrm{H} \cdots \mathrm{O}$ and $\mathrm{C}-$ $\mathrm{H} \cdots \pi$ (arene) hydrogen bonds

Marisiddaiah Girisha, Hemmige S. Yathirajan, Ravindranath S. Rathore and Christopher

\section{Glidewell}

Computing details

Data collection: APEX2 (Bruker, 2012); cell refinement: SAINT (Bruker, 2012); data reduction: SAINT (Bruker, 2012); program(s) used to solve structure: SHELXT2014 (Sheldrick, 2015a); program(s) used to refine structure: SHELXL2014 (Sheldrick, 2015b); molecular graphics: PLATON (Spek, 2009); software used to prepare material for publication:

SHELXL2014 (Sheldrick, 2015b) and PLATON (Spek, 2009).

$N$-(4-chlorobenzylidene)-2-hydroxyaniline

Crystal data

$\mathrm{C}_{13} \mathrm{H}_{10} \mathrm{ClNO}$

$M_{r}=231.67$

Monoclinic, $P 2{ }_{1} / n$

$a=13.0830(17) \AA$

$b=5.8746(6) \AA$

$c=14.825(2) \AA$

$\beta=101.521(4)^{\circ}$

$V=1116.5(2) \AA^{3}$

$Z=4$

Data collection

Bruker APEXII

diffractometer

Radiation source: fine focus sealed tube

Graphite monochromator

$\varphi$ and $\omega$ scans

Absorption correction: multi-scan

(SADABS; Bruker, 2012)

$T_{\min }=0.897, T_{\max }=0.957$

Refinement

Refinement on $F^{2}$

Least-squares matrix: full

$R\left[F^{2}>2 \sigma\left(F^{2}\right)\right]=0.042$

$w R\left(F^{2}\right)=0.106$

$S=1.02$

2565 reflections
$F(000)=480$

$D_{\mathrm{x}}=1.378 \mathrm{Mg} \mathrm{m}^{-3}$

Mo $K \alpha$ radiation, $\lambda=0.71073 \AA$

Cell parameters from 2571 reflections

$\theta=2.3-27.6^{\circ}$

$\mu=0.32 \mathrm{~mm}^{-1}$

$T=296 \mathrm{~K}$

Block, brown

$0.24 \times 0.22 \times 0.14 \mathrm{~mm}$

11962 measured reflections

2565 independent reflections

1556 reflections with $I>2 \sigma(I)$

$R_{\text {int }}=0.032$

$\theta_{\max }=27.5^{\circ}, \theta_{\min }=2.3^{\circ}$

$h=-16 \rightarrow 16$

$k=-7 \rightarrow 7$

$l=-18 \rightarrow 19$

148 parameters

0 restraints

Hydrogen site location: mixed

$\mathrm{H}$ atoms treated by a mixture of independent

and constrained refinement 
$w=1 /\left[\sigma^{2}\left(F_{\mathrm{o}}^{2}\right)+(0.0322 P)^{2}+0.4436 P\right]$

where $P=\left(F_{\mathrm{o}}^{2}+2 F_{\mathrm{c}}{ }^{2}\right) / 3$

$(\Delta / \sigma)_{\max }<0.001$

$$
\Delta \rho_{\max }=0.16 \mathrm{e} \AA^{-3}
$$

\section{Special details}

Geometry. All esds (except the esd in the dihedral angle between two 1.s. planes) are estimated using the full covariance matrix. The cell esds are taken into account individually in the estimation of esds in distances, angles and torsion angles; correlations between esds in cell parameters are only used when they are defined by crystal symmetry. An approximate (isotropic) treatment of cell esds is used for estimating esds involving 1.s. planes.

Fractional atomic coordinates and isotropic or equivalent isotropic displacement parameters $\left(\AA^{2}\right)$

\begin{tabular}{|c|c|c|c|c|}
\hline & $x$ & $y$ & $z$ & $U_{\text {iso }} * / U_{\text {eq }}$ \\
\hline $\mathrm{C} 1$ & $0.65441(13)$ & $0.6411(3)$ & $0.62783(12)$ & $0.0374(4)$ \\
\hline $\mathrm{C} 2$ & $0.68202(15)$ & $0.8448(3)$ & $0.59096(13)$ & $0.0423(5)$ \\
\hline $\mathrm{O} 2$ & $0.60543(11)$ & $0.9939(2)$ & $0.55382(11)$ & $0.0604(4)$ \\
\hline $\mathrm{H} 2$ & 0.5507 (19) & $0.928(5)$ & $0.5621(18)$ & $0.091 *$ \\
\hline $\mathrm{C} 3$ & $0.78503(15)$ & $0.8963(4)$ & $0.59195(14)$ & $0.0494(5)$ \\
\hline H3 & 0.8029 & 1.0334 & 0.5678 & $0.059 *$ \\
\hline $\mathrm{C} 4$ & $0.86113(15)$ & $0.7427(4)$ & $0.62911(14)$ & $0.0523(5)$ \\
\hline H4 & 0.9307 & 0.7753 & 0.6290 & $0.063 *$ \\
\hline $\mathrm{C} 5$ & $0.83542(15)$ & $0.5410(4)$ & $0.66645(14)$ & $0.0500(5)$ \\
\hline H5 & 0.8876 & 0.4392 & 0.6921 & $0.060 *$ \\
\hline C6 & $0.73255(14)$ & 0.4898 & $0.66596(13)$ & $0.0436(5)$ \\
\hline H6 & 0.7155 & 0.3535 & 0.6912 & $0.052 *$ \\
\hline N1 & $0.54588(11)$ & $0.6144(3)$ & $0.62148(10)$ & $0.0422(4)$ \\
\hline C11 & $0.39586(13)$ & 0.3955 & $0.63857(12)$ & $0.0390(4)$ \\
\hline $\mathrm{C} 12$ & $0.32219(14)$ & 0.5524 & $0.59676(13)$ & $0.0452(5)$ \\
\hline H12 & 0.3437 & 0.6879 & 0.5740 & $0.054 *$ \\
\hline C13 & $0.21690(15)$ & $0.5086(4)$ & $0.58864(14)$ & $0.0507(5)$ \\
\hline $\mathrm{H} 13$ & 0.1675 & 0.6142 & 0.5609 & $0.061 *$ \\
\hline C14 & $0.18611(14)$ & $0.3071(4)$ & $0.62218(14)$ & $0.0479(5)$ \\
\hline $\mathrm{Cl14}$ & $0.05422(4)$ & $0.24857(13)$ & $0.60947(5)$ & $0.0861(3)$ \\
\hline C15 & $0.25681(16)$ & $0.1493(4)$ & $0.66378(14)$ & $0.0520(5)$ \\
\hline H15 & 0.2347 & 0.0140 & 0.6863 & $0.062 *$ \\
\hline $\mathrm{C} 16$ & $0.36183(15)$ & $0.1950(3)$ & $0.67172(14)$ & $0.0496(5)$ \\
\hline H16 & 0.4106 & 0.0889 & 0.6999 & $0.059 *$ \\
\hline $\mathrm{C} 17$ & $0.50747(14)$ & $0.4332(3)$ & $0.64610(13)$ & $0.0429(5)$ \\
\hline H17 & 0.5529 & 0.3169 & 0.6705 & $0.052 *$ \\
\hline
\end{tabular}

Atomic displacement parameters $\left(\AA^{2}\right)$

\begin{tabular}{lllllll}
\hline & $U^{11}$ & $U^{22}$ & $U^{33}$ & $U^{12}$ & $U^{13}$ & $U^{23}$ \\
\hline C1 & $0.0388(9)$ & $0.0405(10)$ & $0.0329(10)$ & $0.0007(8)$ & $0.0071(8)$ & $-0.0036(8)$ \\
C2 & $0.0476(11)$ & $0.0400(11)$ & $0.0391(11)$ & $0.0006(9)$ & $0.0086(8)$ & $-0.0013(9)$ \\
O2 & $0.0563(9)$ & $0.0476(9)$ & $0.0756(11)$ & $0.0059(7)$ & $0.0095(8)$ & $0.0162(8)$ \\
C3 & $0.0549(12)$ & $0.0458(12)$ & $0.0498(12)$ & $-0.0093(10)$ & $0.0160(10)$ & $-0.0007(10)$ \\
C4 & $0.0403(10)$ & $0.0644(14)$ & $0.0540(13)$ & $-0.0073(10)$ & $0.0139(9)$ & $-0.0068(11)$ \\
C5 & $0.0405(11)$ & $0.0565(13)$ & $0.0525(13)$ & $0.0062(10)$ & $0.0081(9)$ & $0.0010(11)$
\end{tabular}


supporting information

\begin{tabular}{lllllll} 
C6 & $0.0430(10)$ & $0.0431(11)$ & $0.0448(11)$ & $0.0009(9)$ & $0.0085(8)$ & $0.0024(9)$ \\
N1 & $0.0386(8)$ & $0.0434(9)$ & $0.0440(10)$ & $0.0007(7)$ & $0.0068(7)$ & $0.0020(8)$ \\
C11 & $0.0388(10)$ & $0.0414(11)$ & $0.0364(10)$ & $0.0008(8)$ & $0.0064(8)$ & $-0.0022(9)$ \\
C12 & $0.0460(11)$ & $0.0401(11)$ & $0.0489(12)$ & $-0.0011(9)$ & $0.0084(9)$ & $0.0016(9)$ \\
C13 & $0.0448(11)$ & $0.0493(12)$ & $0.0571(13)$ & $0.0076(10)$ & $0.0078(9)$ & $0.0029(10)$ \\
C14 & $0.0408(10)$ & $0.0549(13)$ & $0.0503(12)$ & $-0.0055(9)$ & $0.0149(9)$ & $-0.0070(10)$ \\
C11 & $0.0454(3)$ & $0.1019(5)$ & $0.1145(6)$ & $-0.0124(3)$ & $0.0244(3)$ & $0.0021(4)$ \\
C15 & $0.0574(13)$ & $0.0454(12)$ & $0.0558(13)$ & $-0.0091(10)$ & $0.0178(10)$ & $0.0015(10)$ \\
C16 & $0.0486(11)$ & $0.0451(12)$ & $0.0534(13)$ & $0.0018(9)$ & $0.0064(9)$ & $0.0098(10)$ \\
C17 & $0.0396(10)$ & $0.0414(11)$ & $0.0461(12)$ & $0.0048(8)$ & $0.0042(8)$ & $0.0021(9)$ \\
\hline
\end{tabular}

Geometric parameters $\left(\AA,{ }^{\circ}\right)$

\begin{tabular}{|c|c|c|c|}
\hline $\mathrm{C} 1-\mathrm{C} 6$ & $1.387(2)$ & $\mathrm{C} 11-\mathrm{C} 16$ & $1.384(3)$ \\
\hline $\mathrm{C} 1-\mathrm{C} 2$ & $1.393(3)$ & $\mathrm{C} 11-\mathrm{C} 12$ & $1.387(2)$ \\
\hline $\mathrm{C} 1-\mathrm{N} 1$ & $1.413(2)$ & $\mathrm{C} 11-\mathrm{C} 17$ & $1.459(2)$ \\
\hline $\mathrm{C} 2-\mathrm{O} 2$ & $1.361(2)$ & $\mathrm{C} 12-\mathrm{C} 13$ & $1.383(3)$ \\
\hline $\mathrm{C} 2-\mathrm{C} 3$ & $1.379(3)$ & $\mathrm{C} 12-\mathrm{H} 12$ & 0.9300 \\
\hline $\mathrm{O} 2-\mathrm{H} 2$ & $0.84(3)$ & $\mathrm{C} 13-\mathrm{C} 14$ & $1.375(3)$ \\
\hline $\mathrm{C} 3-\mathrm{C} 4$ & $1.375(3)$ & $\mathrm{C} 13-\mathrm{H} 13$ & 0.9300 \\
\hline $\mathrm{C} 3-\mathrm{H} 3$ & 0.9300 & $\mathrm{C} 14-\mathrm{C} 15$ & $1.366(3)$ \\
\hline $\mathrm{C} 4-\mathrm{C} 5$ & $1.377(3)$ & $\mathrm{C} 14-\mathrm{Cl14}$ & $1.7324(19)$ \\
\hline $\mathrm{C} 4-\mathrm{H} 4$ & 0.9300 & $\mathrm{C} 15-\mathrm{C} 16$ & $1.382(3)$ \\
\hline $\mathrm{C} 5-\mathrm{C} 6$ & $1.378(3)$ & $\mathrm{C} 15-\mathrm{H} 15$ & 0.9300 \\
\hline $\mathrm{C} 5-\mathrm{H} 5$ & 0.9300 & $\mathrm{C} 16-\mathrm{H} 16$ & 0.9300 \\
\hline $\mathrm{C} 6-\mathrm{H} 6$ & 0.9300 & $\mathrm{C} 17-\mathrm{H} 17$ & 0.9300 \\
\hline $\mathrm{N} 1-\mathrm{C} 17$ & $1.262(2)$ & & \\
\hline $\mathrm{C} 6-\mathrm{C} 1-\mathrm{C} 2$ & $118.87(17)$ & $\mathrm{C} 16-\mathrm{C} 11-\mathrm{C} 17$ & $119.35(17)$ \\
\hline $\mathrm{C} 6-\mathrm{C} 1-\mathrm{N} 1$ & $127.23(18)$ & $\mathrm{C} 12-\mathrm{C} 11-\mathrm{C} 17$ & $121.94(17)$ \\
\hline $\mathrm{C} 2-\mathrm{C} 1-\mathrm{N} 1$ & $113.90(16)$ & $\mathrm{C} 13-\mathrm{C} 12-\mathrm{C} 11$ & 120.45 (19) \\
\hline $\mathrm{O} 2-\mathrm{C} 2-\mathrm{C} 3$ & $120.15(18)$ & $\mathrm{C} 13-\mathrm{C} 12-\mathrm{H} 12$ & 119.8 \\
\hline $\mathrm{O} 2-\mathrm{C} 2-\mathrm{C} 1$ & $118.93(17)$ & $\mathrm{C} 11-\mathrm{C} 12-\mathrm{H} 12$ & 119.8 \\
\hline $\mathrm{C} 3-\mathrm{C} 2-\mathrm{C} 1$ & $120.92(17)$ & $\mathrm{C} 14-\mathrm{C} 13-\mathrm{C} 12$ & $119.16(18)$ \\
\hline $\mathrm{C} 2-\mathrm{O} 2-\mathrm{H} 2$ & $103.0(18)$ & $\mathrm{C} 14-\mathrm{C} 13-\mathrm{H} 13$ & 120.4 \\
\hline $\mathrm{C} 4-\mathrm{C} 3-\mathrm{C} 2$ & $119.24(19)$ & $\mathrm{C} 12-\mathrm{C} 13-\mathrm{H} 13$ & 120.4 \\
\hline $\mathrm{C} 4-\mathrm{C} 3-\mathrm{H} 3$ & 120.4 & $\mathrm{C} 15-\mathrm{C} 14-\mathrm{C} 13$ & $121.77(18)$ \\
\hline $\mathrm{C} 2-\mathrm{C} 3-\mathrm{H} 3$ & 120.4 & $\mathrm{C} 15-\mathrm{C} 14-\mathrm{Cl14}$ & $118.96(17)$ \\
\hline $\mathrm{C} 3-\mathrm{C} 4-\mathrm{C} 5$ & $120.68(18)$ & $\mathrm{C} 13-\mathrm{C} 14-\mathrm{Cl14}$ & $119.26(16)$ \\
\hline $\mathrm{C} 3-\mathrm{C} 4-\mathrm{H} 4$ & 119.7 & $\mathrm{C} 14-\mathrm{C} 15-\mathrm{C} 16$ & $118.58(19)$ \\
\hline $\mathrm{C} 5-\mathrm{C} 4-\mathrm{H} 4$ & 119.7 & $\mathrm{C} 14-\mathrm{C} 15-\mathrm{H} 15$ & 120.7 \\
\hline $\mathrm{C} 4-\mathrm{C} 5-\mathrm{C} 6$ & $120.16(19)$ & $\mathrm{C} 16-\mathrm{C} 15-\mathrm{H} 15$ & 120.7 \\
\hline $\mathrm{C} 4-\mathrm{C} 5-\mathrm{H} 5$ & 119.9 & $\mathrm{C} 15-\mathrm{C} 16-\mathrm{C} 11$ & $121.36(18)$ \\
\hline $\mathrm{C} 6-\mathrm{C} 5-\mathrm{H} 5$ & 119.9 & $\mathrm{C} 15-\mathrm{C} 16-\mathrm{H} 16$ & 119.3 \\
\hline $\mathrm{C} 5-\mathrm{C} 6-\mathrm{C} 1$ & $120.12(19)$ & $\mathrm{C} 11-\mathrm{C} 16-\mathrm{H} 16$ & 119.3 \\
\hline $\mathrm{C} 5-\mathrm{C} 6-\mathrm{H} 6$ & 119.9 & $\mathrm{~N} 1-\mathrm{C} 17-\mathrm{C} 11$ & $123.79(17)$ \\
\hline $\mathrm{C} 1-\mathrm{C} 6-\mathrm{H} 6$ & 119.9 & $\mathrm{~N} 1-\mathrm{C} 17-\mathrm{H} 17$ & 118.1 \\
\hline $\mathrm{C} 17-\mathrm{N} 1-\mathrm{C} 1$ & $121.83(16)$ & $\mathrm{C} 11-\mathrm{C} 17-\mathrm{H} 17$ & 118.1 \\
\hline
\end{tabular}




$\begin{array}{ll}\mathrm{C} 16-\mathrm{C} 11-\mathrm{C} 12 & 118.69(17) \\ \mathrm{C} 6-\mathrm{C} 1-\mathrm{C} 2-\mathrm{O} 2 & -179.76(17) \\ \mathrm{N} 1-\mathrm{C} 1-\mathrm{C} 2-\mathrm{O} 2 & 0.1(2) \\ \mathrm{C} 6-\mathrm{C} 1-\mathrm{C} 2-\mathrm{C} 3 & 0.1(3) \\ \mathrm{N} 1-\mathrm{C} 1-\mathrm{C} 2-\mathrm{C} 3 & 179.96(17) \\ \mathrm{O} 2-\mathrm{C} 2-\mathrm{C} 3-\mathrm{C} 4 & -179.48(18) \\ \mathrm{C} 1-\mathrm{C} 2-\mathrm{C} 3-\mathrm{C} 4 & 0.7(3) \\ \mathrm{C} 2-\mathrm{C} 3-\mathrm{C} 4-\mathrm{C} 5 & -1.1(3) \\ \mathrm{C} 3-\mathrm{C} 4-\mathrm{C} 5-\mathrm{C} 6 & 0.8(3) \\ \mathrm{C} 4-\mathrm{C} 5-\mathrm{C} 6-\mathrm{C} 1 & 0.0(3) \\ \mathrm{C} 2-\mathrm{C} 1-\mathrm{C} 6-\mathrm{C} 5 & -0.4(3) \\ \mathrm{N} 1-\mathrm{C} 1-\mathrm{C} 6-\mathrm{C} 5 & 179.72(18) \\ \mathrm{C} 6-\mathrm{C} 1-\mathrm{N} 1-\mathrm{C} 17 & -4.9(3) \\ \mathrm{C} 2-\mathrm{C} 1-\mathrm{N} 1-\mathrm{C} 17 & 175.25(17) \\ \end{array}$

$$
\begin{aligned}
& \mathrm{C} 16-\mathrm{C} 11-\mathrm{C} 12-\mathrm{C} 13 \\
& \mathrm{C} 17-\mathrm{C} 11-\mathrm{C} 12-\mathrm{C} 13 \\
& \mathrm{C} 11-\mathrm{C} 12-\mathrm{C} 13-\mathrm{C} 14 \\
& \mathrm{C} 12-\mathrm{C} 13-\mathrm{C} 14-\mathrm{C} 15 \\
& \mathrm{C} 12-\mathrm{C} 13-\mathrm{C} 14-\mathrm{C} 114 \\
& \mathrm{C} 13-\mathrm{C} 14-\mathrm{C} 15-\mathrm{C} 16 \\
& \mathrm{C} 114-\mathrm{C} 14-\mathrm{C} 15-\mathrm{C} 16 \\
& \mathrm{C} 14-\mathrm{C} 15-\mathrm{C} 16-\mathrm{C} 11 \\
& \mathrm{C} 12-\mathrm{C} 11-\mathrm{C} 16-\mathrm{C} 15 \\
& \mathrm{C} 17-\mathrm{C} 11-\mathrm{C} 16-\mathrm{C} 15 \\
& \mathrm{C} 1-\mathrm{N} 1-\mathrm{C} 17-\mathrm{C} 11 \\
& \mathrm{C} 16-\mathrm{C} 11-\mathrm{C} 17-\mathrm{N} 1 \\
& \mathrm{C} 12-\mathrm{C} 11-\mathrm{C} 17-\mathrm{N} 1
\end{aligned}
$$$$
-0.3(3)
$$$$
0.4(3)
$$$$
-178.35(15)
$$$$
-0.2(3)
$$$$
178.52(16)
$$$$
0.0(3)
$$$$
0.1(3)
$$$$
-178.19(18)
$$$$
-178.31(16)
$$$$
-176.83(18)
$$$$
5.0(3)
$$

Hydrogen-bond geometry $\left(A,{ }^{\circ}\right)$

$\mathrm{Cg} 1$ and $\mathrm{Cg} 2$ are the centroids of the $\mathrm{C} 1-\mathrm{C} 6$ and $\mathrm{C} 11-\mathrm{C} 16$ rings, respectively.

\begin{tabular}{lllll}
\hline$D-\mathrm{H} \cdots A$ & $D-\mathrm{H}$ & $\mathrm{H} \cdots A$ & $D \cdots A$ & $D-\mathrm{H} \cdots A$ \\
\hline $\mathrm{O} 2-\mathrm{H} 2 \cdots \mathrm{N} 1$ & $0.84(3)$ & $2.05(3)$ & $2.626(2)$ & $125(2)$ \\
$\mathrm{O} 2-\mathrm{H} 2 \cdots \mathrm{O} 2^{\mathrm{i}}$ & $0.84(3)$ & $2.44(3)$ & $2.899(2)$ & $115(2)$ \\
$\mathrm{C} 6-\mathrm{H} 6 \cdots C g 1^{\mathrm{ii}}$ & 0.93 & 2.79 & $3.491(2)$ & 133 \\
$\mathrm{C} 15-\mathrm{H} 15 \cdots C g 2^{\mathrm{iii}}$ & 0.93 & 2.96 & $3.675(2)$ & 135 \\
\hline
\end{tabular}

Symmetry codes: (i) $-x+1,-y+2,-z+1$; (ii) $-x+3 / 2, y-1 / 2,-z+3 / 2$; (iii) $-x+1 / 2, y-1 / 2,-z+3 / 2$. 